\title{
Bendamustin in der Primärtherapie
}

\author{
Prof. Dr. Wolfgang Knauf, Frankfurt/ \\ Main, stellte Studienergebnissen der \\ Studiengruppe indolente Lymphome \\ (STiL) zu Bendamustin bei Patienten \\ mit indolenten Non-Hodgkin-Lym- \\ phomen (iNHL) vor.
}

Bei Patienten mit iNHL hat sich als Primärtherapie die Kombination von Bendamustin (Levact $^{\circledR}$ ) mit Rituximab (B-R) im direkten Vergleich mit der kombinierten Immunochemotherapie mit Rituximab (CHOP [Cyclophosphamid/Hydroxydaunorubicin/Vincristin/Predniso(lo)n]-R) als ebenso wirksam erwiesen (Rummel MJ et al., 2008 Blood 112:\#2596; Rummel MJ et al., 2009 Blood 114:\#405 und oral presentation; Rummel MJ et al., 2012 J Clin Oncol 30 [Suppl ]:\#3).

\section{Verbessertes PFS}

Mit einem Gesamtansprechen (ORR) von 92,7\% bzw. 91,3\% (B-R bzw. CHOP-R) und
Komplettremissions-Raten (CR) von 39,6\% bzw. 30,0\% $(\mathrm{p}=0,0262)$ konnten in beiden Therapiearmen ähnliche Ergebnisse erzielt werden. Auch das mediane progressionsfreie Überleben (PFS) verbesserte sich unter B-R bei Patienten mit follikulärem Lymphom (FL) und Morbus Waldenström deutlich. Bei dem sonst aggressiver behandelten Mantelzell-Lymphom (MCL) konnten ebenfalls gute Ergebnisse mit einer ORR von $89 \%$ unter B-R im Vergleich zu 95\% unter CHOP-R und einer CR von 32\% bzw. 35\% erreicht werden. Bemerkenswert war auch, dass unter B-R eine Verlängerung des PFS von mehr einem Jahr beobachtet wurde (35,4 versus 22,1 Monate).

Der Einfluss der B-R-Therapie auf die Mobilisierbarkeit von Stammzellen der Patienten wurde ebenfalls untersucht. Zwar "kann B-R kein Mobilisierungsschema sein", so warnte Knauf, aber auch nach B-R konnten Stammzellen gewonnen werden.

\section{Alopezie wird nicht induziert}

Vor allem die Alopezie ist für Patienten eine emotionale Belastung. Diese wurde durch die Behandlung mit B-R nicht induziert. Parästhesien und Leukozytopenien traten deutlich seltener auf. Insgesamt korrelierte B-R im Vergleich zu CHOP-R mit einer geringeren Hämatotoxizität, jedoch wurden Erytheme häufiger beobachtet $(\mathrm{p}=0,0122)$ sowie allergische Reaktionen der Haut $(p=0,0003)$. Neben dem Therapiestandard CHOP-R wurde auch B-R in die Leitlinien der Deutschen Gesellschaft für Hämatologie und Onkologie e.V. (DGHO) für die Primärtherapie bei Patienten mit iNHL aufgenommen. Es sollte die bevorzugte Erstlinientherapie für Patienten mit FL, iNHL und MCL sein und ,in Studien der neue Vergleichstherapiearm“, schlussfolgerte Knauf.

Jubiläums-Symposium der Mundipharma GmbH

\section{Optimale Sequenztherapie ermöglicht langes Überleben}

Die Kombination Bevacizumab plus Interferon a (IFN a) hat sich seit 2007 als effektive First-line-Therapie bei Patienten mit metastasiertem Nierenzellkarzinom (mRCC) bewährt. Experten empfehlen den Einsatz in der Erstlinie vor allem bei Patienten mit guter Prognose und/oder indolenter Erkrankung. Denn bei einer optimalen Sequenztherapie können später weitere Substanzen mit anderem Wirkmechanismus eingesetzt werden - der Patient profitiert mit einem langen progressionsfreien Überleben und Gesamtüberleben.

Zur Behandlung des mRCC wurden innerhalb kurzer Zeit mehrere Biologics zugelassen, die die Prognose der Patienten deutlich verbesserten: „Im Vergleich zur Zytokin-Ära konnte das Überleben verdoppelt werden“, konstatierte Prof. Dr. Jan Roigas, Berlin. Allerdings gibt es für die zielgerichtete Therapie beim mRCC noch keine molekularen Prädiktoren, welche Substanz für welchen $\mathrm{Pa}$ tienten am besten geeignet ist. Vielmehr muss man Patienten anhand klinischer Kriterien für eine definierte Therapie selektieren.

\section{Klinische Kriterien für Therapie- wahl nutzen}

Um mRCC-Patienten ein langes Überleben zu sichern, sollte laut Roigas eine optimale Sequenztherapie angestrebt werden. Bei Patienten mit der günstigen Risikokategorie und indolenter Erkrankung, d.h. langsamem Progress, asymptomatischen Metastasen und gutem Allgemeinzustand, die etwa 30$40 \%$ der nicht vorbehandelten mRCC-Patienten ausmachen, sollte bevorzugt die Kombination Bevacizumab/IFN a gewählt werden. Sie hat neben ihrer in Studien belegten hohen Effektivität den Vorteil der guten Verträglichkeit. Damit lassen sich Patienten gut für die Behandlung motivieren und halten diese auch langfristig durch. Zudem stehen bei Progress alle weiteren Substanzklassen - d.h. Tyrosinkinase-Inhibitoren und mTOR (mammalian Target of Rapamycin)Inhibitoren - für die Sequenztherapie zur Verfügung. Letztlich kann der Patient so über einen langen Zeitraum behandelt und ihm ein langes Überleben ermöglicht werden.

Somit hat ein Paradigmenwechsel stattgefunden, konstatierte Roigas: Der Therapieeinstieg mit der potentesten, zumeist aber auch toxischen Substanz, sonst ein Dogma in der Onkologie, ist bei Patienten mit mRCC nicht unbedingt angebracht. Wenn ein besser verträglicher Wirkstoff in der Erstlinientherapie ebenfalls gut wirksam ist, bietet das den zusätzlichen Vorteil, die körperlichen Reserven des Patienten zu schonen und ihm so die Möglichkeit auf weitere Therapien zu bieten. „Mit dem Regime Bevacizumab (Avastin $\left.{ }^{\circledR}\right) /$ IFN a können wir für den Patienten auf hohem Niveau in puncto Lebensqualität Zeit gewinnen“, resümierte Roigas.

(arn)

Anmerkung der Redaktion: Wie eine SubgruppenanaIyse der Studie AVOREN und die Phase-II-Studie BEVLin ergaben, können die Nebenwirkungen durch Reduktion der IFN a-Dosis ohne Wirkverlust der Kombinationstherapie verringert werden (Melichar B et al., Ann Oncol 2008, 19:1470-1476 und ESMO 2012:\#809P).

Pressekonferenz der Roche Pharma AG 